\title{
A linear algebra approach to the hybrid Sheffer-Appell polynomials
}

\author{
Subuhi Khan ${ }^{1} \cdot$ Mahvish Ali ${ }^{1}$
}

Received: 5 September 2017 / Accepted: 17 April 2019 / Published online: 25 April 2019

(c) The Author(s) 2019

\begin{abstract}
In this article, a method based on linear algebra approach is adopted to study the hybrid Sheffer-Appell polynomials. The recursive formulas and differential equation for these polynomials are derived by using the properties and relationships between the Pascal functional matrices and the Wronskian matrices. The corresponding results for some mixed type special polynomials are also obtained.
\end{abstract}

Keywords Sheffer polynomials - Generalized Pascal functional matrix $\cdot$ Wronskian matrix $\cdot$ Recursive formulas

Differential equation

Mathematics Subject Classification $15 \mathrm{~A} 15 \cdot 15 \mathrm{~A} 24 \cdot 33 \mathrm{C} 45 \cdot 65 \mathrm{QXX}$

\section{Introduction and preliminaries}

Recently, a systematic study of certain new classes of mixed special polynomials related to the Appell and Sheffer polynomial sequences is introduced and studied, see for example [3-7]. These mixed special polynomials are important due to the fact that they posses important properties such as differential equations, generating functions, series definitions, integral representations etc. The problems arising in different areas of science and engineering are usually expressed in terms of differential equations, which in most of the cases have special functions as their solutions. Recently, Srivastava et al. [12] derived the differential, integro-differential and partial differential equations for the Hermite-based Appell polynomials [7] using the factorization method. Further, the same method is extended by Khan and Riyasat [5] to derive a set of differential equations of finite order for the 2-iterated Appell polynomials [3]. In this article, recursive formulas and differential equation for the hybrid Sheffer-Appell polynomials are derived by applying the algebra of Pascal and Wronskian matrices.

Mahvish Ali

mahvishali37@gmail.com

Subuhi Khan

subuhi2006@gmail.com

1 Department of Mathematics, Aligarh Muslim University, Aligarh, India
We review some definitions and concepts related to the Pascal and Wronskian matrices which will be used in Sects. 2 and 3.

Let $\mathcal{F}=\left\{h(t)=\sum_{k=0}^{\infty} a_{k} \frac{t^{k}}{k !} \mid a_{k} \in \mathbb{C}\right\}$ be the $\mathbb{C}$-algebra of formal power series.

For $h(t) \in \mathcal{F}$, the generalized Pascal functional matrix [13] of an analytic function $h(t)$ denoted by $\mathcal{P}_{n}[h(t)]$ is a square matrix of order $(n+1)$ defined as :

$\mathcal{P}_{n}[h(t)]_{i, j}= \begin{cases}\left(\begin{array}{l}i \\ j\end{array}\right) h^{(i-j)}(t), & \text { if } i \geq j, \quad i, j=0,1,2, \ldots, n \\ 0, & \text { otherwise. }\end{cases}$

It should be noted that $h^{(k)}$ denotes the $k$ th order derivative of $h$ and $h^{k}$ denotes the $k$ th power of $h$ throughout the article.

Also, the $n$th order Wronskian matrix of analytic functions $h_{1}(t), h_{2}(t), h_{3}(t), \ldots, h_{m}(t)$ is an $(n+1) \times m$ matrix and is defined as:

$$
\begin{aligned}
\mathcal{W}_{n} & {\left[h_{1}(t), h_{2}(t), h_{3}(t), \ldots, h_{m}(t)\right] } \\
& =\left[\begin{array}{ccccc}
h_{1}(t) & h_{2}(t) & h_{3}(t) & \cdots & h_{m}(t) \\
h_{1}^{\prime}(t) & h_{2}^{\prime}(t) & h_{3}^{\prime}(t) & \cdots & h_{m}^{\prime}(t) \\
\vdots & \vdots & \vdots & \ddots & \vdots \\
h_{1}^{(n)}(t) & h_{2}^{(n)}(t) & h_{3}^{(n)}(t) & \cdots & h_{m}^{(n)}(t)
\end{array}\right] .
\end{aligned}
$$


It is important to note that in the expressions $\mathcal{P}_{n}[h(x, t)]_{t=0}$ and $\mathcal{W}_{n}[h(x, t)]_{t=0}$, we consider $t$ as the working variable and $x$ as a parameter.

We recall certain important properties and relationships between the Pascal functional and Wronskian matrices [14].

For any $a, b \in \mathbb{C}$ and any analytic functions $h(t), l(t) \in \mathcal{F}$, the following properties hold true:

$$
\begin{aligned}
& \mathcal{P}_{n}[a h(t)+b l(t)]=a \mathcal{P}_{n}[h(t)]+b \mathcal{P}_{n}[l(t)] . \\
& \mathcal{W}_{n}[a h(t)+b l(t)]=a \mathcal{W}_{n}[h(t)]+b \mathcal{W}_{n}[l(t)] . \\
& \mathcal{P}_{n}[l(t)] \mathcal{P}_{n}[h(t)]=\mathcal{P}_{n}[h(t)] \mathcal{P}_{n}[l(t)]=\mathcal{P}_{n}[h(t) l(t)] . \\
& \mathcal{P}_{n}[l(t)] \mathcal{W}_{n}[h(t)]=\mathcal{P}_{n}[h(t)] \mathcal{W}_{n}[l(t)]=\mathcal{W}_{n}[h(t) l(t)] . \\
& \mathcal{W}_{n}[l(h(t))]_{t=0}=\mathcal{W}_{n}\left[1, h(t), h^{2}(t), h^{3}(t),\right. \\
& \left.\quad \ldots, h^{n}(t)\right]_{t=0} \Lambda_{n}^{-1} \mathcal{W}_{n}[l(t)]_{t=0},
\end{aligned}
$$

where $\Lambda_{n}=\operatorname{diag}[0 !, 1 !, 2 !, \ldots, n !]$ and $h(0)=0$ and $h^{\prime}(0) \neq 0$.

Further, for any analytic functions $l(t)$ and $h_{1}(t), h_{2}(t), \ldots, h_{m}(t)$, the following property holds true:

$$
\begin{aligned}
\mathcal{P}_{n} & {[l(t)] \mathcal{W}_{n}\left[h_{1}(t), h_{2}(t), \ldots, h_{m}(t)\right] } \\
& =\mathcal{W}_{n}\left[\left(l h_{1}\right)(t),\left(l h_{2}\right)(t), \ldots,\left(l h_{m}\right)(t)\right] .
\end{aligned}
$$

One of the important classes of polynomial sequences is the class of Appell polynomial sequences [1]. The Appell polynomials constitute an important class of polynomials because of their remarkable applications in numerous fields. The Appell polynomials appear in different applications in pure and applied mathematics. These polynomials arise in theoretical physics, chemistry and several branches of mathematics such as the study of polynomial expansions of analytic functions, number theory and numerical analysis.

In 1880, Appell [1] introduced and studied sequences of $n$-degree polynomials $A_{n}(x), n=0,1,2, \ldots$, satisfying the recurrence relation

$\frac{\mathrm{d}}{\mathrm{d} x} A_{n}(x)=n A_{n-1}(x), \quad n=1,2, \ldots$

The generating function of the sequence of polynomials $A_{n}(x)$ is given as:

$\frac{e^{x t}}{a(t)}=\sum_{n=0}^{\infty} A_{n}(x) \frac{t^{n}}{n !}$,

where

$a(t)=\sum_{n=0}^{\infty} \aleph_{n} \frac{t^{n}}{n !}, \quad \aleph_{0} \neq 0$.

Another, important class of polynomial sequences is the class of Sheffer sequences. The Sheffer sequences [11] arise in numerous problems of applied mathematics, theoretical physics, approximation theory and several other mathematical branches. According to Roman [10], the Sheffer sequence $s_{n}(x)$ is uniquely determined by two (formal) power series:

$f(t)=\sum_{n=0}^{\infty} f_{n} \frac{t^{n}}{n !}, \quad f_{0}=0, f_{1} \neq 0$

and

$\mathrm{g}(t)=\sum_{n=0}^{\infty} \mathrm{g}_{n} \frac{t^{n}}{n !}, \mathrm{g}_{0} \neq 0$

Then, the exponential generating function of $s_{n}(x)$ is given by:

$\frac{e^{x f^{-1}(t)}}{\mathrm{g}\left(f^{-1}(t)\right)}=\sum_{n=0}^{\infty} s_{n}(x) \frac{t^{n}}{n !}$,

for all $x$ in $\mathbb{C}$, where $f^{-1}(t)$ is the compositional inverse of $f(t)$.

It should be noted that for $\mathrm{g}(t)=1$, the Sheffer sequence $s_{n}(x)$ becomes the associated Sheffer sequence $\mathfrak{S}_{n}(x)$ and for $f(t)=t$, it becomes the Appell sequence $A_{n}(x)$.

Certain members belonging to the Sheffer associated Sheffer and Appell families are given in the Table 1.

By combining the Sheffer and Appell sequences, an extended class of Sheffer polynomials namely the Sheffer-Appell polynomials denoted by ${ }_{s} A_{n}(x)$ is introduced and studied in [4], this class is defined by the following generating function:

$\frac{e^{x f^{-1}(t)}}{\mathrm{g}\left(f^{-1}(t)\right) a(t)}=\sum_{n=0}^{\infty}{ }_{s} A_{n}(x) \frac{t^{n}}{n !}$,

where $\mathrm{g}(t), a(t)$ are the invertible series with $\mathrm{g}(0) \neq 0, a(0) \neq 0$ and $f(t)$ is a delta series with $f(0)=0$ and $f^{\prime}(0) \neq 0$.

We remark that since the 1.h.s. of definition (15) includes $\mathrm{g}(t), f(t)$ of Sheffer class, while $a(t)$ in the denominator is corresponding to the Appell class. Therefore, it is justified to call this hybrid family as Sheffer-Appell family. The main advantage of this family is that it allows to consider mixed type special polynomials by taking $\mathrm{g}(t), f(t)$ of the members belonging to the Sheffer family and $a(t)$ of the members belonging to the Appell family.

In [14], Youn and Yang derived the differential equation and recursive formulas for the Sheffer polynomial sequences using the generalized Pascal functional matrix of an analytic function and Wronskian matrix of several analytic functions. In this article, the method adopted in [14] is extended to derive the recursive formulas and differential 
Table 1 Certain members of the Sheffer, associated Sheffer and Appell families

Sheffer polynomials

\begin{tabular}{llll}
\hline S. no. & $\mathrm{g}(t) ; f(t) ; f^{-1}(t)$ & Generating function & Polynomials \\
\hline I. & $e^{\frac{t^{2}}{4}} ; \frac{t}{2} ; 2 t$ & $e^{2 x t-t^{2}}=\sum_{n=0}^{\infty} H_{n}(x) \frac{t^{n}}{n !}$ & Hermite polynomials $H_{n}(x)[9]$ \\
II. & $(1-t)^{-1} ; \frac{t}{t-1} ; \frac{t}{t-1}$ & $\frac{1}{(1-t)} \exp \left(\frac{x t}{t-1}\right)=\sum_{n=0}^{\infty} L_{n}(x) t^{n}$ & Laguerre polynomials $n ! L_{n}(x)[9]$
\end{tabular}

Associated Sheffer polynomials

\begin{tabular}{llll}
\hline S. no. & $f(t) ; f^{-1}(t)$ & Generating function & Polynomials \\
\hline I. & $\ln (1+t) ; e^{t}-1$ & $\exp \left(x\left(e^{t}-1\right)\right)=\sum_{n=0}^{\infty} \phi_{n}(x) \frac{t^{n}}{n !}$ & Exponential polynomials $\phi_{n}(x)$ [2] \\
II. & $-\frac{1}{2} t^{2}+t ; 1-\sqrt{1-2 t}$ & $\exp (x(1-\sqrt{1-2 t}))=\sum_{n=0}^{\infty} p_{n}(x) \frac{t^{n}}{n !}$ & Bessel polynomials $p_{n}(x)[8]$ \\
\hline
\end{tabular}

Appell polynomials

\begin{tabular}{llll}
\hline S. no. & $a(t)$ & Generating function & Polynomials \\
\hline I. & $\frac{e^{t}-1}{t}$ & $\frac{t}{\left(e^{t}-1\right)} e^{x t}=\sum_{n=0}^{\infty} B_{n}(x) \frac{t^{n}}{n !}$ & Bernoulli polynomials $B_{n}(x)[9]$ \\
II. & $\frac{e^{t}+1}{2}$ & $\frac{2}{\left(e^{t}+1\right)} e^{x t}=\sum_{n=0}^{\infty} E_{n}(x) \frac{t^{n}}{n !}$ & Euler polynomials $E_{n}(x)[9]$ \\
\hline
\end{tabular}

equation satisfied by the Sheffer-Appell polynomials ${ }_{s} A_{n}(x)$. In Sect. 2, some recursive formulas satisfied by ${ }_{s} A_{n}(x)$ are established. In Sect. 3, differential equation for the Sheffer-Appell polynomials is derived. Certain examples are considered in Sect. 4.

\section{Recursive formulas}

In order to utilize the Wronskian matrices, the vector form of the Sheffer-Appell polynomial sequence is required.

The Sheffer-Appell vector denoted by ${ }_{s} \overline{\mathcal{A}}_{n}(x)$ is defined as:

$\bar{s}_{s}(x)=\left[{ }_{s} A_{0}(x){ }_{s} A_{1}(x){ }_{s} A_{2}(x) \quad \ldots \quad{ }_{s} A_{n}(x)\right]^{\mathrm{T}}$,

where $\left\{{ }_{s} A_{n}(x)\right\}$ is the Sheffer-Appell polynomials sequence defined by Eq. (15).

Since $\frac{e^{x f^{-1}(t)}}{\mathrm{g}\left(f^{-1}(t)\right) a(t)}$ is analytic, therefore by Taylor's theorem, it follows that

${ }_{s} A_{k}(x)=\left.\left(\frac{\mathrm{d}}{\mathrm{d} t}\right)^{k} \frac{e^{x f^{-1}(t)}}{\mathrm{g}\left(f^{-1}(t)\right) a(t)}\right|_{t=0}, \quad k \geq 0$.

In view of Eq. (17), the Sheffer-Appell vector (16) can be expressed as:

$$
\begin{aligned}
\bar{s}_{s}(x) & =\left[{ }_{s} A_{0}(x){ }_{s} A_{1}(x){ }_{s} A_{2}(x) \ldots{ }_{s} A_{n}(x)\right]^{\mathrm{T}} \\
& =\mathcal{W}_{n}\left[\frac{e^{x f^{-1}(t)}}{\mathrm{g}\left(f^{-1}(t)\right) a(t)}\right]_{t=0} .
\end{aligned}
$$

First, we prove the following Lemma:
Lemma 2.1 Let $\left\{{ }_{s} A_{n}(x)\right\}$ be the Sheffer-Appell polynomial sequence. Then,

$$
\begin{aligned}
\left.\left(\mathcal{W}_{n}{ }_{s} A_{0}(x),{ }_{s} A_{1}(x),{ }_{s} A_{2}(x), \ldots,{ }_{s} A_{n}(x)\right]\right)^{\mathrm{T}} \Lambda_{n}^{-1} \\
=\mathcal{P}_{n}\left[\frac{1}{a(t)}\right]_{t=0} \mathcal{W}_{n}\left[1, f^{-1}(t),\left(f^{-1}(t)\right)^{2}\right. \\
\left.\quad \ldots,\left(f^{-1}(t)\right)^{n}\right]_{t=0} \times \Lambda_{n}^{-1} \mathcal{P}_{n}\left[\frac{1}{\mathrm{~g}(t)}\right]_{t=0} \mathcal{P}_{n}\left[e^{x t}\right]_{t=0}
\end{aligned}
$$

Proof By making use of property (6) in the r.h.s. of Eq. (18) and then using property (7) in the second term on r.h.s. of the resultant equation, it follows that

$$
\begin{aligned}
{ }_{s} A_{0}(x) & \left.{ }_{s} A_{1}(x){ }_{s} A_{2}(x) \ldots{ }_{s} A_{n}(x)\right]^{\mathrm{T}} \\
= & \mathcal{P}_{n}\left[\frac{1}{a(t)}\right]_{t=0} \mathcal{W}_{n}\left[1, f^{-1}(t),\left(f^{-1}(t)\right)^{2}, \ldots,\left(f^{-1}(t)\right)^{n}\right]_{t=0} \\
& \times \Lambda_{n}^{-1} \mathcal{W}_{n}\left[\frac{e^{x t}}{\mathrm{~g}(t)}\right]_{t=0} .
\end{aligned}
$$

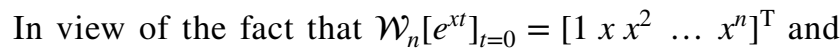
using property (6), Eq. (20) takes the form

$$
\begin{aligned}
{ }_{s} A_{0}(x) & \left.{ }_{s} A_{1}(x){ }_{s} A_{2}(x) \ldots{ }_{s} A_{n}(x)\right]^{\mathrm{T}} \\
= & \mathcal{P}_{n}\left[\frac{1}{a(t)}\right]_{t=0} \mathcal{W}_{n}\left[1, f^{-1}(t),\left(f^{-1}(t)\right)^{2}, \ldots,\left(f^{-1}(t)\right)^{n}\right]_{t=0} \\
& \times \Lambda_{n}^{-1} \mathcal{P}_{n}\left[\frac{1}{\mathrm{~g}(t)}\right]_{t=0}\left[\begin{array}{llllll}
1 & x & x^{2} & \ldots & x^{n}
\end{array}\right]^{\mathrm{T}}
\end{aligned}
$$


Differentiation of Eq. (21) $k$ times with respect to $x$ and division by $k$ ! gives

$$
\begin{aligned}
& \frac{1}{k !}\left[{ }_{s} A_{0}^{(k)}(x){ }_{s} A_{1}^{(k)}(x) \ldots{ }_{s} A_{n}^{(k)}(x)\right]^{\mathrm{T}} \\
& =\mathcal{P}_{n}\left[\frac{1}{a(t)}\right]_{t=0} \mathcal{W}_{n}\left[1, f^{-1}(t),\left(f^{-1}(t)\right)^{2}, \ldots,\left(f^{-1}(t)\right)^{n}\right]_{t=0} \Lambda_{n}^{-1} \\
& \times \mathcal{P}_{n}\left[\frac{1}{\mathrm{~g}(t)}\right]_{t=0}\left[\begin{array}{llll}
0 & \cdots & 0 & 1
\end{array}\left(\begin{array}{c}
k+1 \\
k
\end{array}\right) x\left(\begin{array}{c}
k+2 \\
k
\end{array}\right) x^{2} \ldots\left(\begin{array}{l}
n \\
k
\end{array}\right) x^{n-k}\right]^{\mathrm{T}} .
\end{aligned}
$$

The 1.h.s. of Eq. (22) is the $k$ th column of

$$
\left(\mathcal{W}_{n}\left[{ }_{s} A_{0}(x){ }_{s} A_{1}(x){ }_{s} A_{2}(x) \quad \ldots{ }_{s} A_{n}^{(k)}(x)\right]\right)^{\mathrm{T}} \Lambda_{n}^{-1}
$$

and the r.h.s. of Eq. (22) is the $k$ th column of

$$
\begin{aligned}
\mathcal{P}_{n}\left[\frac{1}{a(t)}\right]_{t=0} \mathcal{W}_{n}\left[1, f^{-1}(t),\left(f^{-1}(t)\right)^{2},\right. \\
\left.\quad \ldots,\left(f^{-1}(t)\right)^{n}\right]_{t=0} \Lambda_{n}^{-1} \mathcal{P}_{n}\left[\frac{1}{\mathrm{~g}(t)}\right]_{t=0} \mathcal{P}_{n}\left[e^{x t}\right]_{t=0} .
\end{aligned}
$$

Hence, assertion (19) follows.

Now, we establish certain recursive formulas for the Sheffer-Appell polynomials ${ }_{s} A_{n}(x)$.

First, we derive a recursive formula, which expresses ${ }_{s} A_{n+1}(x)$ in terms of ${ }_{s} A_{n}(x)$ and its derivatives by proving the following result:

Theorem 2.1 Let ${ }_{s} A_{n}(x)$ denote the Sheffer-Appell polynomials. Then ${ }_{s} A_{0}(x)=\frac{1}{\mathrm{~g}(0) a(0)}$ and

${ }_{s} A_{n+1}(x)=\sum_{k=0}^{n}\left(\delta_{k} x+\eta_{k}+\xi_{k}\right) \frac{{ }_{s} A_{n}^{(k)}(x)}{k !}, \quad n \geq 0$,

where

$$
\begin{aligned}
\delta_{k} & =\left.\left(\frac{1}{f^{\prime}(t)}\right)^{(k)}\right|_{t=0} ; \eta_{k}=\left.\left(-\frac{\mathrm{g}^{\prime}(t)}{\mathrm{g}(t) f^{\prime}(t)}\right)^{(k)}\right|_{t=0} ; \xi_{k} \\
& =\left.\left(-\frac{a^{\prime}(f(t))}{a(f(t))}\right)^{(k)}\right|_{t=0} .
\end{aligned}
$$

Proof In view of definition (2) and Eq. (17), we have

$$
\begin{aligned}
\mathcal{W}_{n} & {\left[\frac{\mathrm{d}}{\mathrm{d} t}\left(\frac{e^{x f^{-1}(t)}}{\mathrm{g}\left(f^{-1}(t)\right) a(t)}\right)\right]_{t=0} } \\
& \left.={ }_{s} A_{1}(x){ }_{s} A_{2}(x){ }_{s} A_{3}(x) \ldots{ }_{s} A_{n+1}(x)\right]^{\mathrm{T}} .
\end{aligned}
$$

Performing the differentiation in expression $\mathcal{W}_{n}\left[\frac{\mathrm{d}}{\mathrm{d} t}\left(\frac{e^{x f^{-1}(t)}}{\mathrm{g}\left(f^{-1}(t)\right) a(t)}\right)\right]_{t=0}$ and using properties (5)-(7) in a suitable manner, we have

$$
\begin{aligned}
\mathcal{W}_{n}[ & \left.\frac{\mathrm{d}}{\mathrm{d} t}\left(\frac{e^{x f^{-1}(t)}}{\mathrm{g}\left(f^{-1}(t)\right) a(t)}\right)\right]_{t=0} \\
= & \mathcal{W}_{n}\left[\left(x-\frac{\mathrm{g}^{\prime}\left(f^{-1}(t)\right)}{\mathrm{g}\left(f^{-1}(t)\right)}\right) \frac{e^{x f^{-1}(t)}}{a(t) f^{\prime}\left(f^{-1}(t)\right) \mathrm{g}\left(f^{-1}(t)\right)}\right. \\
& \left.-\frac{a^{\prime}(t) e^{x f^{-1}(t)}}{(a(t))^{2} \mathrm{~g}\left(f^{-1}(t)\right)}\right]_{t=0} \\
= & \mathcal{P}_{n}\left[\frac{1}{a(t)}\right] \mathcal{W}_{n}\left[1, f^{-1}(t),\left(f^{-1}(t)\right)^{2}, \ldots,\left(f^{-1}(t)\right)^{n}\right]_{t=0} \Lambda_{n}^{-1} \\
& \times \mathcal{W}_{n}\left[\left(x-\frac{\mathrm{g}^{\prime}(t)}{\mathrm{g}(t)}\right) \frac{e^{x t}}{f^{\prime}(t) \mathrm{g}(t)}-\frac{a^{\prime}(f(t)) e^{x t}}{a(f(t)) \mathrm{g}(t)}\right]_{t=0} \\
= & \mathcal{P}_{n}\left[\frac{1}{a(t)}\right]_{t=0} \mathcal{W}_{n}\left[1, f^{-1}(t),\left(f^{-1}(t)\right)^{2}, \ldots,\left(f^{-1}(t)\right)^{n}\right]_{t=0} \Lambda_{n}^{-1} \\
& \times \mathcal{P}_{n}\left[\frac{1}{\mathrm{~g}(t)}\right]_{t=0} \mathcal{P}_{n}\left[e^{x t}\right]_{t=0} \mathcal{W}_{n}\left[\frac{x}{f^{\prime}(t)}-\frac{\mathrm{g}^{\prime}(t)}{\mathrm{g}(t) f^{\prime}(t)}-\frac{a^{\prime}(f(t))}{a(f(t))}\right]_{t=0} .
\end{aligned}
$$

Further, in view of Lemma 2.1, we have

$$
\begin{aligned}
& \mathcal{W}_{n}\left[\frac{\mathrm{d}}{\mathrm{d} t}\left(\frac{e^{x f^{-1}(t)}}{\mathrm{g}\left(f^{-1}(t)\right) a(t)}\right)\right]_{t=0} \\
& =\left(\mathcal{W}_{n}\left[{ }_{s} A_{0}(x),{ }_{s} A_{1}(x),{ }_{s} A_{2}(x), \ldots,{ }_{s} A_{n}(x)\right]\right)^{\mathrm{T}} \Lambda_{n}^{-1} \\
& \times \mathcal{W}_{n}\left[\frac{x}{f^{\prime}(t)}-\frac{\mathrm{g}^{\prime}(t)}{\mathrm{g}(t) f^{\prime}(t)}-\frac{a^{\prime}(f(t))}{a(f(t))}\right]_{t=0} \\
& =\left[\begin{array}{ccccc}
{ }_{s} A_{0}(x) & 0 & 0 & \cdots & 0 \\
{ }_{s} A_{1}(x) & \frac{{ }_{s} A_{1}^{\prime}(x)}{1 !} & 0 & \cdots & 0 \\
{ }_{s} A_{2}(x) & \frac{{ }_{s} A_{2}^{\prime}(x)}{1 !} & \frac{{ }_{s} A_{2}^{\prime \prime}(x)}{2 !} & \cdots & 0 \\
\vdots & \vdots & \vdots & \ddots & \vdots \\
{ }_{s} A_{n}(x) & \frac{{ }_{s} A_{n}^{\prime}(x)}{1 !} & \frac{{ }_{s} A_{n}^{\prime \prime}(x)}{2 !} & \cdots & \frac{{ }_{s} A_{n}^{(n)}(x)}{n !}
\end{array}\right]\left[\begin{array}{c}
\delta_{0} x+\eta_{0}+\xi_{0} \\
\delta_{1} x+\eta_{1}+\xi_{1} \\
\vdots \\
\delta_{n} x+\eta_{n}+\xi_{n}
\end{array}\right] .
\end{aligned}
$$

Equating the last rows of Eqs. (24) and (26), we get assertion (23).

Remark 2.1 Since $\mathrm{g}(t)=1 \Longrightarrow \eta_{k}=0(k \geq 0)$, therefore for $\mathrm{g}(t)=1$, we deduce the following consequence of Theorem 2.1:

Corollary 2.1 Let ${ }_{3} A_{n}(x)$ denote the associated ShefferAppell polynomials. Then ${ }_{\mathfrak{S}} A_{0}(x)=\frac{1}{a(0)}$ and

$$
{ }_{\mathfrak{s}} A_{n+1}(x)=\sum_{k=0}^{n}\left(\delta_{k} x+\xi_{k}\right) \frac{{ }_{\mathfrak{s}} A_{n}^{(k)}(x)}{k !}, \quad n \geq 0,
$$


where

$\delta_{k}=\left.\left(\frac{1}{f^{\prime}(t)}\right)^{(k)}\right|_{t=0} ; \xi_{k}=\left.\left(-\frac{a^{\prime}(f(t))}{a(f(t))}\right)^{(k)}\right|_{t=0}$.

Remark 2.2 Since $f(t)=t \Longrightarrow \delta_{0}=1 ; \delta_{k}=0(k \neq 0)$, therefore for $f(t)=t$, we deduce the following consequence of Theorem 2.1:

Corollary 2.2 Let $A_{n}^{[2]}(x)$ denote the 2-iterated Appell polynomials. Then $A_{0}^{[2]}(x)=\frac{1}{\mathrm{~g}(0) a(0)}$ and

$A_{n+1}^{[2]}(x)=x A_{n}^{[2]}(x)+\sum_{k=0}^{n}\left(\sigma_{k}+\rho_{k}\right) \frac{\left(A_{n}^{[2]}(x)\right)^{(k)}(x)}{k !}, \quad n \geq 0$,

where

$\sigma_{k}=\left.\left(-\frac{\mathrm{g}^{\prime}(t)}{\mathrm{g}(t)}\right)^{(k)}\right|_{t=0} ; \rho_{k}=\left.\left(-\frac{a^{\prime}(t)}{a(t)}\right)^{(k)}\right|_{t=0}$

Next, we derive a pure recurrence relation which expresses ${ }_{s} A_{n+1}(x)$ in terms of ${ }_{s} A_{k}(x)(k=0,1, \ldots, n)$ in the form of following theorem:

Theorem 2.2 Let ${ }_{s} A_{n}(x)$ denotes the Sheffer-Appell polynomials. Then ${ }_{s} A_{0}(x)=\frac{1}{\mathrm{~g}(0) a(0)}$ and

$$
\begin{aligned}
\epsilon_{0} A_{s+1}(x)= & x_{s} A_{n}(x)+\sum_{k=0}^{n}\left(\begin{array}{l}
n \\
k
\end{array}\right)\left(\theta_{k}+v_{k}\right)_{s} A_{n-k}(x) \\
& -\sum_{k=1}^{n}\left(\begin{array}{l}
n \\
k
\end{array}\right) \epsilon_{k s} A_{n+1-k}(x), \quad n \geq 0,
\end{aligned}
$$

where

$$
\begin{gathered}
\epsilon_{k}=\left.\left(f^{\prime}\left(f^{-1}(t)\right)\right)^{(k)}\right|_{t=0}=\left.\left(\frac{1}{\left(f^{-1}(t)\right)^{\prime}}\right)^{(k)}\right|_{t=0} ; \\
\theta_{k}=\left.\left(-\frac{\mathrm{g}^{\prime}\left(f^{-1}(t)\right)}{\mathrm{g}\left(f^{-1}(t)\right)}\right)^{(k)}\right|_{t=0} ; \\
v_{k}=\left.\left(-f^{\prime}\left(f^{-1}(t)\right) \frac{a^{\prime}(t)}{a(t)}\right)^{(k)}\right|_{t=0} .
\end{gathered}
$$

Proof Using property (6) in expression $\mathcal{W}_{n}\left[f^{\prime}\left(f^{-1}(t)\right)\right.$ $\left.\frac{\mathrm{d}}{\mathrm{d} t}\left(\frac{e^{x\left(f^{-1}(t)\right)}}{\mathrm{g}\left(f^{-1}(t)\right) a(t)}\right)\right]_{t=0}$, it follows that

$$
\begin{aligned}
\mathcal{W}_{n}\left[f^{\prime}\left(f^{-1}(t)\right) \frac{\mathrm{d}}{\mathrm{d} t}\left(\frac{e^{x\left(f^{-1}(t)\right)}}{\mathrm{g}\left(f^{-1}(t)\right) a(t)}\right)\right]_{t=0} \\
=\mathcal{P}_{n}\left[\frac{\mathrm{d}}{\mathrm{d} t}\left(\frac{e^{x\left(f^{-1}(t)\right)}}{\mathrm{g}\left(f^{-1}(t)\right) a(t)}\right)\right]_{t=0} \mathcal{W}_{n}\left[f^{\prime}\left(f^{-1}(t)\right)\right]_{t=0} \\
=\left[\begin{array}{cccc}
{ }_{s} A_{1}(x) & 0 & \cdots & 0 \\
{ }_{s} A_{2}(x) & { }_{s} A_{1}(x) & \cdots & 0 \\
{ }_{s} A_{3}(x) & \left(\begin{array}{c}
2 \\
1
\end{array}\right){ }_{s} A_{2}(x) & \cdots & 0 \\
\vdots & \vdots & \ddots & \vdots \\
{ }_{s} A_{n+1}(x) & \left(\begin{array}{c}
n \\
1
\end{array}\right){ }_{s} A_{n}(x) & \cdots & { }_{s} A_{1}(x)
\end{array}\right]\left[\begin{array}{c}
\epsilon_{0} \\
\epsilon_{1} \\
\epsilon_{2} \\
\vdots \\
\epsilon_{n}
\end{array}\right] .
\end{aligned}
$$

On the other hand, performing the differentiation in the same expression and using properties (4) and (6), it follows that

$$
\begin{aligned}
& \mathcal{W}_{n}\left[f^{\prime}\left(f^{-1}(t)\right) \frac{\mathrm{d}}{\mathrm{d} t}\left(\frac{e^{x\left(f^{-1}(t)\right)}}{\mathrm{g}\left(f^{-1}(t)\right) a(t)}\right)\right]_{t=0} \\
& =\mathcal{W}_{n}\left[\frac{x e^{x f^{-1}(t)}}{\mathrm{g}\left(f^{-1}(t)\right) a(t)}-\frac{\mathrm{g}^{\prime}\left(f^{-1}(t)\right) e^{x f^{-1}(t)}}{\mathrm{g}\left(f^{-1}(t)\right)^{2} a(t)}\right. \\
& \left.-f^{\prime}\left(f^{-1}(t)\right) \frac{a^{\prime}(t) e^{x f^{-1}(t)}}{a(t)^{2} \mathrm{~g}\left(f^{-1}(t)\right)}\right]_{t=0} \\
& =x \mathcal{W}_{n}\left[\frac{e^{x\left(f^{-1}(t)\right)}}{\mathrm{g}\left(f^{-1}(t)\right) a(t)}\right]_{t=0}+\mathcal{P}_{n}\left[\frac{e^{x\left(f^{-1}(t)\right)}}{\mathrm{g}\left(f^{-1}(t)\right) a(t)}\right]_{t=0} \\
& \times \mathcal{W}_{n}\left[-\frac{\mathrm{g}^{\prime}\left(f^{-1}(t)\right)}{\mathrm{g}\left(f^{-1}(t)\right)}-f^{\prime}\left(f^{-1}(t)\right) \frac{a^{\prime}(t)}{a(t)}\right]_{t=0} \\
& =x\left[\begin{array}{c}
{ }_{s} A_{0}(x) \\
{ }_{s} A_{1}(x) \\
{ }_{s} A_{2}(x) \\
\vdots \\
{ }_{s} A_{n}(x)
\end{array}\right]+\left[\begin{array}{cccc}
{ }_{s} A_{0}(x) & 0 & \cdots & 0 \\
{ }_{s} A_{1}(x) & { }_{s} A_{0}(x) & \cdots & 0 \\
{ }_{s} A_{2}(x) & \left(\begin{array}{c}
2 \\
1
\end{array}\right){ }_{s} A_{1}(x) & \cdots & 0 \\
\vdots & \vdots & \ddots & \vdots \\
{ }_{s} A_{n}(x) & \left(\begin{array}{c}
n \\
1
\end{array}\right){ }_{s} A_{n-1}(x) & \cdots & { }_{s} A_{0}(x)
\end{array}\right] \\
& \times\left[\begin{array}{c}
\theta_{0}+v_{0} \\
\theta_{1}+v_{1} \\
\theta_{2}+v_{2} \\
\vdots \\
\theta_{n}+v_{n}
\end{array}\right] .
\end{aligned}
$$


Equating the last rows of Eqs. (30) and (31), we get assertion (29)

Remark 2.3 Since $\mathrm{g}(t)=1 \Longrightarrow \theta_{k}=0(k \geq 0)$, therefore for $\mathrm{g}(t)=1$, we deduce the following consequence of Theorem 2.2:

Corollary 2.3 Let ${ }_{3} A_{n}(x)$ denote the associated ShefferAppell polynomials. Then ${ }_{3} A_{0}(x)=\frac{1}{a(0)}$ and

$$
\begin{aligned}
\epsilon_{0}{ }_{\mathfrak{S}} A_{n+1}(x)=x_{\mathfrak{S}} A_{n}(x) \\
\quad+\sum_{k=0}^{n}\left(\begin{array}{l}
n \\
k
\end{array}\right) v_{k} A_{\mathfrak{S}} A_{n-k}(x)-\sum_{k=1}^{n}\left(\begin{array}{l}
n \\
k
\end{array}\right) \epsilon_{k} A_{\mathfrak{S}} A_{n+1-k}(x), \quad n \geq 0,
\end{aligned}
$$

where

$$
\begin{aligned}
\epsilon_{k}=\left.\left(f^{\prime}\left(f^{-1}(t)\right)\right)^{(k)}\right|_{t=0} & =\left.\left(\frac{1}{\left(f^{-1}(t)\right)^{\prime}}\right)^{(k)}\right|_{t=0} ; v_{k} \\
& =\left.\left(-f^{\prime}\left(f^{-1}(t)\right) \frac{a^{\prime}(t)}{a(t)}\right)^{(k)}\right|_{t=0} .
\end{aligned}
$$

Remark 2.4 Since $f(t)=t \Longrightarrow \epsilon_{0}=1 ; \epsilon_{k}=0(k \neq 0)$, therefore for $f(t)=t$, we deduce the following consequence of Theorem 2.2:

Corollary 2.4 Let $A_{n}^{[2]}(x)$ denote the 2-iterated Appell polynomials. Then $A_{0}^{[2]}(x)=\frac{1}{\mathrm{~g}(0) a(0)}$ and

$$
A_{n+1}^{[2]}(x)=x A_{n}^{[2]}(x)+\sum_{k=0}^{n}\left(\begin{array}{l}
n \\
k
\end{array}\right)\left(\chi_{k}+\tau_{k}\right) A_{n-k}^{[2]}(x), \quad n \geq 0,
$$

where

$\chi_{k}=\left.\left(-\frac{\mathrm{g}^{\prime}(t)}{\mathrm{g}(t)}\right)^{(k)}\right|_{t=0} ; \tau_{k}=\left.\left(-\frac{a^{\prime}(t)}{a(t)}\right)^{(k)}\right|_{t=0}$

Finally, we derive a pure recurrence relation, which provides a representation of ${ }_{s} A_{n+1}(x)$ in terms of ${ }_{s} A_{k}(x)$ ( $k=0,1,2, \ldots n)$ by proving the following result:

Theorem 2.3 Let ${ }_{s} A_{n}(x)$ denote the Sheffer-Appell polynomials. Then ${ }_{s} A_{0}(x)=\frac{1}{\mathrm{~g}(0) a(0)}$ and

$$
{ }_{s} A_{n+1}(x)=\sum_{k=0}^{n}\left(\begin{array}{l}
n \\
k
\end{array}\right)\left(x \phi_{k}+\psi_{k}+\lambda_{k}\right)_{s} A_{n-k}(x), \quad n \geq 0,
$$

where

$$
\begin{aligned}
\phi_{k} & =\left.\left(\frac{1}{f^{\prime}\left(f^{-1}(t)\right)}\right)^{(k)}\right|_{t=0} ; \psi_{k} \\
& =\left.\left(-\frac{\mathrm{g}^{\prime}\left(f^{-1}(t)\right)}{\mathrm{g}\left(f^{-1}(t)\right)} \frac{1}{f^{\prime}\left(f^{-1}(t)\right)}\right)^{(k)}\right|_{t=0} ; \lambda_{k}=\left.\left(-\frac{a^{\prime}(t)}{a(t)}\right)^{(k)}\right|_{t=0} .
\end{aligned}
$$

Proof Performing the differentiation in expression $\mathcal{W}_{n}\left[\frac{\mathrm{d}}{\mathrm{d} t}\left(\frac{e^{x f^{-1}(t)}}{\mathrm{g}\left(f^{-1}(t)\right) a(t)}\right)\right]_{t=0}$ and then using property (6), we have

$$
\begin{aligned}
& \mathcal{W}_{n}\left[\frac{\mathrm{d}}{\mathrm{d} t}\left(\frac{e^{x f^{-1}(t)}}{\mathrm{g}\left(f^{-1}(t)\right) a(t)}\right)\right]_{t=0} \\
& =\mathcal{P}_{n}\left[\left(x-\frac{\mathrm{g}^{\prime}\left(f^{-1}(t)\right)}{\mathrm{g}\left(f^{-1}(t)\right)}\right) \frac{1}{f^{\prime}\left(f^{-1}(t)\right)}-\frac{a^{\prime}(t)}{a(t)}\right] \mathcal{W}_{n}\left[\frac{e^{x f^{-1}(t)}}{\mathrm{g}\left(f^{-1}(t)\right) a(t)}\right]_{t=0} \\
& =\left[\begin{array}{ccccc}
x \phi_{0}+\psi_{0}+\lambda_{0} & 0 & 0 & \cdots & 0 \\
x \phi_{1}+\psi_{1}+\lambda_{1} & x \phi_{0}+\psi_{0}+\lambda_{0} & 0 & \cdots & 0 \\
x \phi_{2}+\psi_{2}+\lambda_{2} & \left(\begin{array}{c}
2 \\
1
\end{array}\right) x \phi_{1}+\psi_{1}+\lambda_{1} & x \phi_{0}+\psi_{0}+\lambda_{0} & \cdots & 0 \\
\vdots & \vdots & \vdots & \ddots & \vdots \\
x \phi_{n}+\psi_{n}+\lambda_{n}\left(\begin{array}{c}
n \\
1
\end{array}\right) x \phi_{n-1}+\psi_{n-1}+\lambda_{n-1}\left(\begin{array}{c}
n \\
2
\end{array}\right) x \phi_{n-2}+\psi_{n-2}+\lambda_{n-2} & \cdots & x \phi_{0}+\psi_{0}+\lambda_{0}
\end{array}\right] \\
& \times\left[\begin{array}{c}
{ }_{s} A_{0}(x) \\
{ }_{s} A_{1}(x) \\
{ }_{s} A_{2}(x) \\
\vdots \\
{ }_{s} A_{n}(x)
\end{array}\right] .
\end{aligned}
$$


Equating the last rows of Eqs. (24) and (35), we get assertion (34).

Remark 2.5 Since $\mathrm{g}(t)=1 \Longrightarrow \psi_{k}=0(k \geq 0)$, therefore for $g(t)=1$, we deduce the following consequence of Theorem 2.3:

Corollary 2.5 Let ${ }_{3} A_{n}(x)$ denote the associated ShefferAppell polynomials. Then ${ }_{3} A_{0}(x)=\frac{1}{a(0)}$ and

${ }_{3} A_{n+1}(x)=\sum_{k=0}^{n}\left(\begin{array}{l}n \\ k\end{array}\right)\left(x \phi_{k}+\lambda_{k}\right){ }_{3} A_{n-k}(x), \quad n \geq 0$,

where

$\phi_{k}=\left.\left(\frac{1}{f^{\prime}\left(f^{-1}(t)\right)}\right)^{(k)}\right|_{t=0} ; \lambda_{k}=\left.\left(-\frac{a^{\prime}(t)}{a(t)}\right)^{(k)}\right|_{t=0}$

Remark 2.6 We note that $f(t)=t \Longrightarrow \phi_{0}=1 ; \phi_{k}=0$ $(k \neq 0)$. Therefore, taking $f(t)=t$, in Theorem 2.3, we get Corollary 2.4 .

In the next section, differential equation for the Sheffer-Appell polynomials ${ }_{s} A_{n}(x)$ is derived.

\section{Differential equation}

In order to derive the differential equation for the Sheffer-Appell polynomials ${ }_{s} A_{n}(x)$, we prove the following result:

Theorem 3.1 The Sheffer-Appell polynomials ${ }_{s} A_{n}(x)$ satisfy the following differential equation:

$\sum_{k=1}^{n}\left(\beta_{k} x+\alpha_{k}+\gamma_{k}\right) \frac{{ }_{s} A_{n}^{(k)}(x)}{k !}-n_{s} A_{n}(x)=0$,

where

$$
\begin{aligned}
& \alpha_{k}=\left.\left(-\frac{\mathrm{g}^{\prime}(t) f(t)}{\mathrm{g}(t) f^{\prime}(t)}\right)^{(k)}\right|_{t=0} ; \beta_{k}=\left.\left(\frac{f(t)}{f^{\prime}(t)}\right)^{(k)}\right|_{t=0} ; \\
& \gamma_{k}=\left.\left(-\frac{f(t) a^{\prime}(f(t))}{a(f(t))}\right)^{(k)}\right|_{t=0} .
\end{aligned}
$$

Proof In view of property (6), the expression $\mathcal{W}_{n}\left[t \frac{\mathrm{d}}{\mathrm{d} t}\left(\frac{e^{x f^{-1}(t)}}{\mathrm{g}\left(f^{-1}(t)\right) a(t)}\right)\right]_{t=0}$ can be written as:

$$
\begin{aligned}
& \mathcal{W}_{n}\left[t \frac{\mathrm{d}}{\mathrm{d} t}\left(\frac{e^{x f^{-1}(t)}}{\mathrm{g}\left(f^{-1}(t)\right) a(t)}\right)\right]_{t=0}=\mathcal{P}_{n}[t]_{t=0} \mathcal{W}_{n}\left[\frac{\mathrm{d}}{\mathrm{d} t}\left(\frac{e^{x f^{-1}(t)}}{\mathrm{g}\left(f^{-1}(t)\right) a(t)}\right)\right]_{t=0} \\
& =\left[\begin{array}{cccccccc}
0 & 0 & 0 & 0 & \cdots & 0 & 0 & 0 \\
1 & 0 & 0 & 0 & \cdots & 0 & 0 & 0 \\
0 & 2 & 0 & 0 & \cdots & 0 & 0 & 0 \\
0 & 0 & 3 & 0 & \cdots & 0 & 0 & 0 \\
\vdots & \vdots & \vdots & \ddots & \ddots & \vdots & \vdots & \vdots \\
\ddots & \ddots & & & \\
0 & 0 & 0 & 0 & \cdots & n-1 & 0 & 0 \\
0 & 0 & 0 & 0 & \cdots & 0 & n & 0
\end{array}\right]\left[\begin{array}{c}
{ }_{s} A_{1}(x) \\
{ }_{s} A_{2}(x) \\
{ }_{s} A_{3}(x) \\
\vdots \\
{ }_{s} A_{n}(x) \\
{ }_{s}{ }_{n+1}(x)
\end{array}\right] .
\end{aligned}
$$

On the other hand performing the differentiation in the same expression and using properties (5)-(7) in a suitable manner, we have

$$
\begin{aligned}
\mathcal{W}_{n}[t & \left.\frac{\mathrm{d}}{\mathrm{d} t}\left(\frac{e^{x f^{-1}(t)}}{\mathrm{g}\left(f^{-1}(t)\right) a(t)}\right)\right]_{t=0} \\
= & \mathcal{W}_{n}\left[\left(x-\frac{\mathrm{g}^{\prime}\left(f^{-1}(t)\right)}{\mathrm{g}\left(f^{-1}(t)\right)}\right) \frac{t e^{x f^{-1}(t)}}{a(t) f^{\prime}\left(f^{-1}(t)\right) \mathrm{g}\left(f^{-1}(t)\right)}\right. \\
& \left.-\frac{t a^{\prime}(t) e^{x f^{-1}(t)}}{(a(t))^{2} \mathrm{~g}\left(f^{-1}(t)\right)}\right]_{t=0} \\
= & \mathcal{P}_{n}\left[\frac{1}{a(t)}\right] \mathcal{W}_{n}\left[1, f^{-1}(t),\left(f^{-1}(t)\right)^{2}, \ldots,\left(f^{-1}(t)\right)^{n}\right]_{t=0} \Lambda_{n}^{-1} \\
& \times \mathcal{W}_{n}\left[\left(x-\frac{\mathrm{g}^{\prime}(t)}{\mathrm{g}(t)}\right) \frac{f(t) e^{x t}}{f^{\prime}(t) \mathrm{g}(t)}-\frac{f(t) a^{\prime}(f(t)) e^{x t}}{a(f(t)) \mathrm{g}(t)}\right]_{t=0} \\
= & \mathcal{P}_{n}\left[\frac{1}{a(t)}\right]_{t=0} \mathcal{W}_{n}\left[1, f^{-1}(t),\left(f^{-1}(t)\right)^{2}, \ldots,\left(f^{-1}(t)\right)^{n}\right]_{t=0} \Lambda_{n}^{-1} \\
& \times \mathcal{P}_{n}\left[\frac{1}{\mathrm{~g}(t)}\right]_{t=0} \mathcal{P}_{n}\left[e^{x t}\right]_{t=0} \mathcal{W}_{n}\left[\frac{x f(t)}{f^{\prime}(t)}-\frac{\mathrm{g}^{\prime}(t) f(t)}{\mathrm{g}(t) f^{\prime}(t)}-\frac{f(t) a^{\prime}(f(t))}{a(f(t))}\right]_{t=0} .
\end{aligned}
$$

Again, in view of Lemma 2.1, we have 


$$
\begin{aligned}
\mathcal{W}_{n}\left[t \frac{\mathrm{d}}{\mathrm{d} t}\left(\frac{e^{x f^{-1}(t)}}{\mathrm{g}\left(f^{-1}(t)\right) a(t)}\right)\right]_{t=0}=\left(\mathcal{W}_{n}\left[{ }_{s} A_{0}(x),{ }_{s} A_{1}(x),{ }_{s} A_{2}(x), \ldots,{ }_{s} A_{n}(x)\right]\right)^{\mathrm{T}} \Lambda_{n}^{-1} \\
\times \mathcal{W}_{n}\left[\frac{x f(t)}{f^{\prime}(t)}-\frac{\mathrm{g}^{\prime}(t) f(t)}{\mathrm{g}(t) f^{\prime}(t)}-\frac{f(t) a^{\prime}(f(t))}{a(f(t))}\right]_{t=0} \\
=\left[\begin{array}{ccccc}
{ }_{s} A_{0}(x) & 0 & 0 & \cdots & 0 \\
{ }_{s} A_{1}(x) & \frac{{ }_{s} A_{1}^{\prime}(x)}{1 !} & 0 & \cdots & 0 \\
{ }_{s} A_{2}(x) & \frac{{ }_{s} A_{2}^{\prime}(x)}{1 !} & \frac{{ }_{s} A_{2}^{\prime \prime}(x)}{2 !} & \cdots & 0 \\
\vdots & \vdots & \vdots & \ddots & \vdots \\
{ }_{s} A_{n}(x) & \frac{{ }_{s} A_{n}^{\prime}(x)}{1 !} & \frac{{ }_{s} A_{n}^{\prime \prime}(x)}{2 !} & \cdots & \frac{{ }_{s} A_{n}^{(n)}(x)}{n !}
\end{array}\right]\left[\begin{array}{c}
\beta_{0} x+\alpha_{0}+\gamma_{0} \\
\beta_{1} x+\alpha_{1}+\gamma_{1} \\
\vdots \\
\beta_{n} x+\alpha_{n}+\gamma_{n}
\end{array}\right] .
\end{aligned}
$$

On equating the last rows of Eqs. (38) and (40) and using the fact that

$f(0)=0 \Longrightarrow \alpha_{0}=\beta_{0}=\gamma_{0}=0$,

we get assertion (37).

Remark 3.1 For $\mathrm{g}(t)=1$, the Sheffer-Appell polynomials ${ }_{s} A_{n}(x)$ reduce to the associated Sheffer-Appell polynomials ${ }_{g} A_{n}(x)$ and since $\mathrm{g}(t)=1 \Longrightarrow \alpha_{k}=0(k \geq 1)$, therefore, for $g(t)=1$, we deduce the following consequence of Theorem 3.1:

Corollary 3.1 The associated Sheffer-Appell polynomials ${ }_{5} A_{n}(x)$ satisfy the following differential equation:

$\sum_{k=1}^{n}\left(\beta_{k} x+\gamma_{k}\right) \frac{{ }_{\mathfrak{s}} A_{n}^{(k)}(x)}{k !}-n_{{ }_{3}} A_{n}(x)=0$,

where

$\beta_{k}=\left.\left(\frac{f(t)}{f^{\prime}(t)}\right)^{(k)}\right|_{t=0} ; \gamma_{k}=\left.\left(-\frac{f(t) a^{\prime}(f(t))}{a(f(t))}\right)^{(k)}\right|_{t=0}$.

Remark 3.2 For $f(t)=t$, the Sheffer-Appell polynomials ${ }_{s} A_{n}(x)$ reduce to the 2 -iterated Appell polynomials $A_{n}^{[2]}(x)$ [3] and since $f(t)=t \Longrightarrow \beta_{1}=1 ; \beta_{k}=0(k \neq 1)$, therefore, for $f(t)=t$, we deduce the following consequence of Theorem 3.1:

Corollary 3.2 The 2-iterated Appell polynomials $A_{n}^{[2]}(x)$ satisfy the following differential equation:

$x\left(A_{n}^{[2]}(x)\right)^{\prime}+\sum_{k=1}^{n}\left(\mu_{k}+\omega_{k}\right) \frac{\left(A_{n}^{[2]}(x)\right)^{(k)}}{k !}-n A_{n}^{[2]}(x)=0$,

where $\mu_{k}=\left.\left(-\frac{\operatorname{tg}^{\prime}(t)}{\mathrm{g}(t)}\right)^{(k)}\right|_{t=0} ; \omega_{k}=\left.\left(-\frac{t a^{\prime}(t)}{a(t)}\right)^{(k)}\right|_{t=0}$

In the next section, we obtain the differential equations and recursive formulas for some members belonging to the Sheffer-Appell, associated Sheffer-Appell and 2-iterated Appell families.

\section{Examples}

We derive the differential equations and recursive formulas for some members belonging to the Sheffer-Appell family by applying Theorem 3.1 and Theorems 2.1-2.3, respectively.

Example 4.1 For $\mathrm{g}(t)=e^{\frac{t^{2}}{4}}$ and $f(t)=\frac{t}{2}$, the Sheffer polynomials become the Hermite polynomials $H_{n}(x)$ and for $a(t)=\frac{e^{t}-1}{t}$, the Appell polynomials become the Bernoulli polynomials $B_{n}(x)$. Therefore, for these values of $g(t), f(t)$ and $a(t)$, the Sheffer-Appell polynomials reduce to the Hermite-Bernoulli polynomials ${ }_{H} B_{n}(x)$.

From Theorem 3.1, we find

$\alpha_{k}=\left.\left(-\frac{t^{2}}{2}\right)^{(k)}\right|_{t=0} ; \quad \beta_{k}=\left.(t)^{(k)}\right|_{t=0} ; \quad \gamma_{k}=\left.\left(-\frac{\frac{t}{2} e^{\frac{t}{2}}-e^{\frac{t}{2}}+1}{e^{\frac{t}{2}}-1}\right)^{(k)}\right|_{t=0}$.

Substituting the values from Eq. (43) into Eq. (37), we obtain the following differential equation for the HermiteBernoulli polynomials ${ }_{H} B_{n}(x)$ : 
${ }_{H} B_{n}^{\prime \prime}(x)-2 x_{H} B_{n}^{\prime}(x)-\left.2 \sum_{k=1}^{n}\left(-\frac{\frac{t}{2} e^{\frac{t}{2}}-e^{\frac{t}{2}}+1}{e^{\frac{t}{2}}-1}\right)^{(k)}\right|_{t=0} \frac{{ }_{H} B_{n}^{(k)}(x)}{k !}+2 n_{H} B_{n}(x)=0$.

Similarly, using Theorems 2.1, 2.2 and 2.3, the following recursive formulas for the Hermite-Bernoulli polynomials ${ }_{H} B_{n}(x)$ are obtained:

${ }_{H} B_{0}(x)=1$,

$$
\begin{aligned}
& { }_{H} B_{n+1}(x)=2 x_{H} B_{n}(x)-{ }_{H} B_{n}^{\prime}(x) \\
& \quad+\left.\sum_{k=0}^{n}\left(-\frac{\frac{t}{2} e^{\frac{t}{2}}-e^{\frac{t}{2}}+1}{\frac{t}{2}\left(e^{\frac{t}{2}}-1\right)}\right)^{(k)}\right|_{t=0} \frac{{ }_{H} B_{n}^{(k)}(x)}{k !}, \quad n \geq 0
\end{aligned}
$$

and

$$
\begin{aligned}
& { }_{H} B_{n+1}(x)=2 x_{H} B_{n}(x)-2 n_{H} B_{n-1}(x) \\
& \quad+\left.\sum_{k=0}^{n}\left(\begin{array}{l}
n \\
k
\end{array}\right)\left(-\frac{t e^{t}-e^{t}+1}{t\left(e^{t}-1\right)}\right)^{(k)}\right|_{t=0}{ }_{H} B_{n-k}(x), \quad n \geq 0 .
\end{aligned}
$$

Example 4.2 For $\mathrm{g}(t)=\frac{1}{1-t}$ and $f(t)=\frac{t}{t-1}$, the Sheffer polynomials become the Laguerre polynomials $L_{n}(x)$ and for $a(t)=\frac{e^{t}+1}{2}$, the Appell polynomials become the Euler polynomials $E_{n}(x)$. Therefore, for these values of $\mathrm{g}(t), f(t)$ and $a(t)$, the Sheffer-Appell polynomials reduce to the Laguerre-Euler polynomials ${ }_{L} E_{n}(x)$.

From Theorem 3.1, we find

$\alpha_{k}=\left.(-t)^{(k)}\right|_{t=0} ; \quad \beta_{k}=\left.(-t(t-1))^{(k)}\right|_{t=0} ;$

$\gamma_{k}=\left.\left(-\frac{t e^{\frac{t}{t-1}}}{(t-1)\left(e^{\frac{t}{t-1}}+1\right)}\right)^{(k)}\right|_{t=0}$.

Substituting the values from Eq. (48) into Eq. (37), we obtain the following differential equation for the LaguerreEuler polynomials ${ }_{L} E_{n}(x)$ :

$x_{L} E_{n}^{\prime \prime}(x)-(x-1)_{L} E_{n}^{\prime}(x)-\left.\sum_{k=1}^{n}\left(-\frac{t e^{\frac{t}{t-1}}}{(t-1)\left(e^{\frac{t}{t-1}}+1\right)}\right)^{(k)}\right|_{t=0}$ $\frac{{ }_{L} E_{n}^{(k)}(x)}{k !}+n_{L} E_{n}(x)=0$.
Similarly, using Theorems 2.1, 2.2 and 2.3, the following recursive formulas for the Laguerre-Euler polynomials ${ }_{L} E_{n}(x)$ are obtained:

$$
\begin{aligned}
& { }_{L} E_{0}(x)=1, \\
& { }_{L} E_{n+1}(x)=(1-x)_{L} E_{n}(x)+(2 x-1)_{L} E_{n}^{\prime}(x)-x_{L} E_{n}^{\prime \prime}(x) \\
& \quad+\left.\sum_{k=0}^{n}\left(-\frac{e^{\frac{t}{t-1}}}{e^{\frac{t}{t-1}}+1}\right)^{(k)}\right|_{t=0} \frac{{ }_{L} E_{n}^{(k)}(x)}{k !} \\
& \quad n \geq 0
\end{aligned}
$$

$$
\begin{aligned}
& { }_{L} E_{n+1}(x)=(1-x+2 n)_{L} E_{n}(x)-n^{2}{ }_{L} E_{n-1}(x) \\
& \quad-\left.\sum_{n=0}^{n}\left(\begin{array}{l}
n \\
k
\end{array}\right)\left(\frac{(t-1)^{2} e^{t}}{e^{t}+1}\right)^{(k)}\right|_{t=0}{ }_{L} E_{n-k}(x), \\
& n \geq 0
\end{aligned}
$$

and

$$
\begin{aligned}
& { }_{L} E_{n+1}(x)=\sum_{k=0}^{n} \frac{n !}{(n-k) !}\left((-1)^{k+1}(k+1) x+1\right)_{L} E_{n-k}(x) \\
& \quad+\left.\left(\begin{array}{l}
n \\
k
\end{array}\right)\left(-\frac{e^{t}}{e^{t}+1}\right)^{(k)}\right|_{t=0}{ }_{L} E_{n-k}(x), \\
& \quad n \geq 0 .
\end{aligned}
$$

Next, we apply Corollary 3.1 to derive the differential equations and Corollaries 2.1, 2.3 and 2.5 to derive the recursive formulas for some members belonging to the associated Sheffer-Appell family.

Example 4.3 For $f(t)=\ln (1+t)$, the associated Sheffer polynomials become the exponential polynomials $\phi_{n}(x)$ and for $a(t)=\frac{e^{t}-1}{t}$, the Appell polynomials become the Bernoulli polynomials $B_{n}(x)$. Therefore, for these values of $f(t)$ and $a(t)$, the associated Sheffer-Appell polynomials reduce to the exponential-Bernoulli polynomials ${ }_{\phi} B_{n}(x)$.

From Corollary 3.1, we find

$$
\beta_{k}=\left.((1+t) \ln (1+t))^{(k)}\right|_{t=0} ; \quad \gamma_{k}=\left.\left(\frac{t-(1+t) \ln (1+t)}{t}\right)^{(k)}\right|_{t=0}
$$


From Eqs. (54) and (41), the following differential equation for the exponential-Bernoulli polynomials ${ }_{\phi} B_{n}(x)$ is obtained:

$$
\begin{aligned}
& x_{\phi} B_{n}^{\prime}(x)+x \frac{{ }_{\phi} B_{n}^{\prime \prime}(x)}{2 !}+\sum_{k=3}^{n}(-1)^{(k-2)} x \frac{{ }_{\phi} B_{n}^{(k)}(x)}{k(k-1)} \\
& +\left.\sum_{k=1}^{n}\left(\frac{t-(1+t) \ln (1+t)}{t}\right)^{(k)}\right|_{t=0} \frac{{ }_{\phi} B_{n}^{(k)}(x)}{k !} \\
& -n_{\phi} B_{n}(x)=0 .
\end{aligned}
$$

Similarly, using Corollaries 2.1, 2.3 and 2.5, the following recursive formulas for the exponential-Bernoulli polynomials ${ }_{\phi} B_{n}(x)$ are obtained:

$$
{ }_{\phi} B_{0}(x)=1,
$$

$$
\begin{aligned}
& { }_{\phi} B_{n+1}(x)=x\left({ }_{\phi} B_{n}(x)+{ }_{\phi} B_{n}^{\prime}(x)\right) \\
& +\left.\sum_{k=0}^{n}\left(\frac{t-(1+t) \ln (1+t)}{t \ln (1+t)}\right)^{(k)}\right|_{t=0} \frac{{ }_{\phi} B_{n}^{(k)}(x)}{k !}, \quad n \geq 0,
\end{aligned}
$$

$$
\begin{gathered}
{ }_{\phi} B_{n+1}(x)=x_{\phi} B_{n}(x)-\sum_{k=1}^{n}\left(\begin{array}{l}
n \\
k
\end{array}\right)(-1)_{\phi}^{k} B_{n+1-k}(x) \\
+\left.\sum_{k=0}^{n}\left(\begin{array}{l}
n \\
k
\end{array}\right)\left(\frac{e^{t}-t e^{t}-1}{t e^{t}\left(e^{t}-1\right)}\right)^{(k)}\right|_{t=0}{ }_{\phi} B_{n-k}(x), \\
n \geq 0
\end{gathered}
$$

and

$$
\begin{aligned}
{ }_{\phi} B_{n+1}(x)= & \sum_{k=0}^{n}\left(\begin{array}{l}
n \\
k
\end{array}\right)\left(x+\left.\left(\frac{e^{t}-t e^{t}-1}{t\left(e^{t}-1\right)}\right)^{(k)}\right|_{t=0}\right) \\
& { }_{\phi} B_{n-k}(x), \quad n \geq 0 .
\end{aligned}
$$

Example 4.4 For $f(t)=-\frac{1}{2} t^{2}+t$, the associated Sheffer polynomials become the Bessel polynomials $p_{n}(x)$ and for $a(t)=\frac{e^{t}+1}{2}$, the Appell polynomials become the Euler polynomials $E_{n}(x)$. Therefore, for these values of $f(t)$ and $a(t)$, the associated Sheffer-Appell polynomials reduce to the Bessel-Euler polynomials ${ }_{p} E_{n}(x)$.

From Corollary 3.1, we find

$$
\beta_{k}=\left.\left(\frac{t\left(1-\frac{t}{2}\right)}{1-t}\right)^{(k)}\right|_{t=0} ; \quad \gamma_{k}=\left.\left(-\frac{t\left(1-\frac{t}{2}\right) e^{t\left(1-\frac{t}{2}\right)}}{e^{t\left(1-\frac{t}{2}\right)}+1}\right)^{(k)}\right|_{t=0}
$$

From Eqs. (60) and (41), the following differential equation for the Bessel-Euler polynomials ${ }_{p} E_{n}(x)$ is obtained:

$$
\begin{aligned}
& \sum_{k=1}^{n}\left(\left.x\left(\frac{t\left(1-\frac{t}{2}\right)}{1-t}\right)^{(k)}\right|_{t=0}\right. \\
& \left.\quad+\left.\left(-\frac{t\left(1-\frac{t}{2}\right) e^{t\left(1-\frac{t}{2}\right)}}{e^{t\left(1-\frac{t}{2}\right)}+1}\right)^{(k)}\right|_{t=0}\right) \frac{p E_{n}^{(k)}(x)}{k !}-n_{p} E_{n}(x) .
\end{aligned}
$$

Similarly, using Corollaries 2.1, 2.3 and 2.5, the following recursive formulas for the Bessel-Euler polynomials ${ }_{p} E_{n}(x)$ are obtained:

$$
\begin{aligned}
& { }_{p} E_{0}(x)=1 \\
& { }_{p} E_{n+1}(x)=\sum_{k=0}^{n}\left(k ! x+\left.\left(-\frac{e^{t\left(1-\frac{t}{2}\right)}}{e^{t\left(1-\frac{t}{2}\right)}+1}\right)^{(k)}\right|_{t=0} \frac{{ }_{p} E_{n}^{(k)}(x)}{k !}, \quad n \geq 0\right.
\end{aligned}
$$

$$
\begin{array}{r}
{ }_{p} E_{n+1}(x)=x_{p} E_{n}(x)-\left.\sum_{k=1}^{n}\left(\begin{array}{l}
n \\
k
\end{array}\right)(\sqrt{1-2 t})^{(k)}\right|_{t=0}{ }_{p} E_{n+1-k}(x) \\
+\left.\sum_{k=0}^{n}\left(\begin{array}{l}
n \\
k
\end{array}\right)\left(-\sqrt{1-2 t} \frac{e^{t}}{e^{t}+1}\right)^{(k)}\right|_{t=0} E_{n-k}(x), \quad n \geq 0
\end{array}
$$

and

$$
\begin{aligned}
{ }_{p} E_{n+1}(x)= & \sum_{k=0}^{n}\left(\begin{array}{c}
n \\
k
\end{array}\right)\left(\left.x\left((1-2 t)^{1 / 2}\right)^{(k)}\right|_{t=0}\right. \\
& \left.+\left.\left(-\frac{e^{t}}{e^{t}+1}\right)^{(k)}\right|_{t=0}\right)_{p} E_{n-k}(x), \quad n \geq 0 .
\end{aligned}
$$

Further, we apply Corollary 3.2 to derive the differential equations and Corollaries 2.2 and 2.4 to derive the recursive formulas for some members belonging to the 2-iterated Appell family.

Example 4.5 For $\mathrm{g}(t)=a(t)=\frac{e^{t}-1}{t}$, the 2-iterated Appell polynomials reduce to the 2 -iterated Bernoulli polynomials $B_{n}^{[2]}(x)$.

From Corollary 3.2, we find

$\mu_{k}=\omega_{k}=\left.\left(\frac{e^{t}-t e^{t}-1}{e^{t}-1}\right)^{(k)}\right|_{t=0}$. 
Substituting the values from Eq. (66) in Eq. (42), we find the following differential equation for the 2-iterated Bernoulli polynomials $B_{n}^{[2]}(x)$ :

$$
\begin{gathered}
x\left(B_{n}^{[2]}(x)\right)^{\prime}+\left.2 \sum_{k=1}^{n}\left(\frac{e^{t}-t e^{t}-1}{e^{t}-1}\right)^{(k)}\right|_{t=0} \\
\frac{\left(B_{n}^{[2]}(x)\right)^{(k)}}{k !}-n B_{n}^{[2]}(x)=0 .
\end{gathered}
$$

Similarly, using Corollaries 2.2 and 2.4, the following recursive formulas for the 2-iterated Bernoulli polynomials $B_{n}^{[2]}(x)$ are obtained:

$B_{0}^{[2]}(x)=1$,

$$
\begin{aligned}
& B_{n+1}^{[2]}(x)=x B_{n}^{[2]}(x) \\
& \quad+\left.2 \sum_{k=0}^{n}\left(\begin{array}{l}
n \\
k
\end{array}\right)\left(\frac{e^{t}-t e^{t}-1}{t\left(e^{t}-1\right)}\right)^{(k)}\right|_{t=0} \frac{\left(B_{n}^{[2]}(x)\right)^{(k)}}{k !}, \quad n \geq 0
\end{aligned}
$$

and

$$
\begin{aligned}
B_{n+1}^{[2]}(x)= & x B_{n}^{[2]}(x)+2 \sum_{k=0}^{n}\left(\begin{array}{l}
n \\
k
\end{array}\right) \\
& \left.\left(\frac{e^{t}-t e^{t}-1}{t\left(e^{t}-1\right)}\right)^{(k)}\right|_{t=0} B_{n-k}^{[2]}(x), \quad n \geq 0 .
\end{aligned}
$$

Example 4.6 For $\mathrm{g}(t)=a(t)=\frac{e^{t}+1}{2}$, the 2-iterated Appell polynomials reduce to the 2-iterated Euler polynomials $E_{n}^{[2]}(x)$.

From Corollary 3.2, we find

$\mu_{k}=\omega_{k}=\left.\left(-\frac{t e^{t}}{e^{t}+1}\right)^{(k)}\right|_{t=0}$.

Substituting the values from Eq. (71) into Eq. (42), we find the following differential equation for the 2-iterated Euler polynomials $E_{n}^{[2]}(x)$ :

$x\left(E_{n}^{[2]}(x)\right)^{\prime}+\left.2 \sum_{k=1}^{n}\left(-\frac{t e^{t}}{e^{t}+1}\right)^{(k)}\right|_{t=0} \frac{\left(E_{n}^{[2]}(x)\right)^{(k)}}{k !}-n E_{n}^{[2]}(x)=0$.

Similarly, using Corollaries 2.2 and 2.4, the following recursive formulas for the 2-iterated Euler polynomials $E_{n}^{[2]}(x)$ are obtained:

$$
E_{0}^{[2]}(x)=1
$$

$$
\begin{aligned}
& E_{n+1}^{[2]}(x)=x E_{n}^{[2]}(x) \\
& \quad+\left.2 \sum_{k=0}^{n}\left(\begin{array}{l}
n \\
k
\end{array}\right)\left(-\frac{e^{t}}{e^{t}+1}\right)^{(k)}\right|_{t=0} \frac{\left(E_{n}^{[2]}(x)\right)^{(k)}}{k !}, \quad n \geq 0
\end{aligned}
$$

and

$$
\begin{aligned}
& E_{n+1}^{[2]}(x)=x E_{n}^{[2]}(x) \\
& \quad+\left.2 \sum_{k=0}^{n}\left(\begin{array}{l}
n \\
k
\end{array}\right)\left(-\frac{e^{t}}{e^{t}+1}\right)^{(k)}\right|_{t=0} E_{n-k}^{[2]}(x), \quad n \geq 0 .
\end{aligned}
$$

Example 4.7 For $\mathrm{g}(t)=\frac{e^{t}-1}{t}$ and $a(t)=\frac{e^{t}+1}{2}$, the 2-iterated Appell polynomials reduce to the Bernoulli-Euler polynomials ${ }_{B} E_{n}(x)$.

From Corollary 3.2, we find

$$
\mu_{k}=\left.\left(\frac{e^{t}-t e^{t}-1}{e^{t}-1}\right)^{(k)}\right|_{t=0} ; \quad \omega_{k}=\left.\left(-\frac{t e^{t}}{e^{t}+1}\right)^{(k)}\right|_{t=0}
$$

Substituting the values from Eq. (76) into Eq. (42), the following differential equation for the Bernoulli-Euler polynomials ${ }_{B} E_{n}(x)$ is obtained:

$$
\begin{aligned}
& x_{B} E_{n}^{\prime}(x)+\sum_{k=1}^{n}\left(\left.\left(\frac{e^{t}-t e^{t}-1}{e^{t}-1}\right)^{(k)}\right|_{t=0}\right. \\
& \left.+\left.\left(-\frac{t e^{t}}{e^{t}+1}\right)^{(k)}\right|_{t=0}\right) \frac{{ }_{B} E_{n}^{(k)}(x)}{k !}-n_{B} E_{n}(x)=0 .
\end{aligned}
$$

Similarly, using Corollaries 2.2 and 2.4, the following recursive formulas for the Bernoulli-Euler polynomials ${ }_{B} E_{n}(x)$ are obtained:

${ }_{B} E_{0}(x)=1$,

$$
\begin{aligned}
& { }_{B} E_{n+1}(x)+\sum_{k=0}^{n}\left(\left.\left(\frac{e^{t}-t e^{t}-1}{t\left(e^{t}-1\right)}\right)^{(k)}\right|_{t=0}\right. \\
& \left.+\left.\left(-\frac{t e^{t}}{t\left(e^{t}+1\right)}\right)^{(k)}\right|_{t=0}\right) \frac{{ }_{B} E_{n}^{(k)}(x)}{k !}, \quad n \geq 0
\end{aligned}
$$

and 


$$
\begin{aligned}
{ }_{B} E_{n+1}(x)= & x_{B} E_{n}(x)+\sum_{k=0}^{n}\left(\begin{array}{l}
n \\
k
\end{array}\right)\left(\left.\left(\frac{e^{t}-t e^{t}-1}{t\left(e^{t}-1\right)}\right)^{(k)}\right|_{t=0}\right. \\
& \left.+\left.\left(-\frac{t e^{t}}{t\left(e^{t}+1\right)}\right)^{(k)}\right|_{t=0}\right)_{B} E_{n-k}(x), \\
& n \geq 0 .
\end{aligned}
$$

It is to be noted that the differential equations and recursive formulas for other members belonging to the Sheffer-Appell, associated Sheffer-Appell and 2-iterated Appell families can also be obtained in a similar manner by making suitable substitutions.

In this article, the differential equation and recursive formulas for the Sheffer-Appell polynomial sequences are established by using the Pascal and Wronskian matrices. The corresponding results are also established for the associated Sheffer-Appell and 2-iterated Appell polynomial sequences, which are the subclasses of the Sheffer-Appell polynomial sequences. Since the Sheffer-Appell polynomials are important from the point of view of their applications in various fields, therefore, the differential equation and recursive formulas satisfied by these polynomials may be used to solve the existing as well as new emerging problems in certain branches of science. This approach can be extended to derive the properties of other generalized hybrid special polynomial families.

Acknowledgements This work has been done under Senior Research Fellowship (Award Letter No. F1-17.1/2014-15/MANF-2014-15MUS-UTT-34170/(SA-III/Website)) awarded to the second author by the University Grants Commission, Government of India, New Delhi.

Open Access This article is distributed under the terms of the Creative Commons Attribution 4.0 International License (http://creativeco mmons.org/licenses/by/4.0/), which permits unrestricted use, distribution, and reproduction in any medium, provided you give appropriate credit to the original author(s) and the source, provide a link to the Creative Commons license, and indicate if changes were made.

\section{References}

1. Appell, P.: Sur une classe de polynômes. Ann. Sci. École. Norm. Sup. 9(2), 119-144 (1880)

2. Bell, E.T.: Exponential polynomials. Ann. Math. 35, 258-277 (1934)

3. Khan, S., Raza, N.: 2-Iterated Appell polynomials and related numbers. Appl. Math. Comput. 219, 9469-9483 (2013)

4. Khan, S., Riyasat, M.: A determinantal approach to ShefferAppell polynomials via monomiality principle. J. Math. Anal. Appl. 421, 806-829 (2015)

5. Khan, S., Riyasat, M.: Differential and integral equations for the 2 -iterated Appell polynomials. J. Comput. Appl. Math. 306, 116-132 (2016)

6. Khan, S., Al-Saad, M.W., Khan, R.: Laguerre-based Appell polynomials: properties and applications. Math. Comput. Model. 52(1-2), 247-259 (2010)

7. Khan, S., Yasmin, G., Khan, R., Hassan, N.A.M.: Hermite-based Appell polynomials: properties and applications. J. Math. Anal. Appl 351, 756-764 (2009)

8. Krall, H.L., Frink, O.: A new class of orthogonal polynomials: the Bessel polynomials. Trans. Am. Math. Soc. 65, 100-115 (1949)

9. Rainville, E.D.: Special Functions, Reprint of 1960, 1st edn. Chelsea Publishig, Bronx, New York (1971)

10. Roman, S.: The Umbral Calculus. Academic Press, New York (1984)

11. Sheffer, I.M.: Some properties of polynomial sets of type zero. Duke. Math. J. 5, 590-622 (1939)

12. Srivastav, H.M., Özarslan, M.A., Yilmaz, B.: Some families of differential equations associated with the Hermite-based Appell polynomials and other classes of Hermite-based polynomials. Filomat 28(4), 695-708 (2014)

13. Yang, Y., Micek, C.: Generalized Pascal functional matrix and its applications. Linear Algebra Appl. 423(2-3), 230-245 (2007)

14. Youn, H., Yang, Y.: Differential equation and recursive formulas of Sheffer polynomial sequences. ISRN Discrete Math. 2011, 1-16 (2011). (Article ID 476462)

Publisher's Note Springer Nature remains neutral with regard to jurisdictional claims in published maps and institutional affiliations. 